\title{
Essential oil of Melaleuca alternifolia for the treatment of oral candidiasis induced in an immunosuppressed mouse model
}

\author{
Vanessa Maria de Campos Rasteiro, Anna Carolina Borges Pereira da Costa, Cássia Fernandes Araújo, \\ Patrícia Pimentel de Barros, Rodnei Dennis Rossoni", Ana Lia Anbinder, Antonio Olavo Cardoso Jorge \\ and Juliana Campos Junqueira
}

\begin{abstract}
Background: The search for alternative therapies for oral candidiasis is a necessity and the use of medicinal plants seems to be one of the promising solutions. The objective of this study was to evaluate the in vitro and in vivo effects of the essential oil of Melaleuca alternifolia on Candida albicans.

Methods: The minimum inhibitory concentration (MIC) and minimum biofilm eradication concentration (MBEC) of M. alternifolia were determined by the broth microdilution assay. For the in vivo study, twelve immunosuppressed mice with buccal candidiasis received topical applications of M. alternifolia with MBEC. After treatment, yeasts were recovered from the mice and quantified (CFU/mL). Mice were killed for morphologic analysis of the tongue dorsum by optical and scanning electron microscopy. Data were analyzed using Student's $t$ test or Mann-Whitney test.

Results: The MIC of M. alternifolia was $0.195 \%$ and the MBEC was $12.5 \%$. Treatment with M. alternifolia achieved a 5.33 log reduction in C. albicans and reduced the microscopic lesions of candidiasis.

Conclusions: M. alternifolia oil at a $12.5 \%$ was effective to eradicate a C. albicans biofilm formed in vitro and to reduce yeasts of $C$. albicans in an immunosuppressed mouse model.
\end{abstract}

Keywords: Melaleuca alternifolia, Candida albicans, Oral candidiasis, Murine model

\section{Background}

Recent decades have seen a significant increase in the incidence of all forms of candidiasis. This increase reflects changes in medical practice with more frequent use of invasive surgical procedures, and more widespread use of immunosuppressive therapies and broad-spectrum antibiotics [1]. However, key to the increase in oral candidiasis has been the expansion of acquired immunodeficiency syndrome (AIDS) worldwide [1]. Oral candidiasis is the most common fungal infection in patients with AIDS and it usually indicates the progression of HIV infection [2-4]. Treatment of oral candidiasis in HIV-positive patients is difficult because of recurring episodes, intermittent exposure and continual selection of antifungal

\footnotetext{
* Correspondence: dennisrossoni@hotmail.com

Department of Biosciences and Oral Diagnosis, Institute of Science and Technology, UNESP - Univ Estadual Paulista, Francisco José Longo 777, São Dimas, São José dos Campos CEP: 12245-000, SP, Brazil
}

therapy-resistant strains. Studies have shown that C. albicans and non-albicans strains are developing resistance to the antifungals widely used for treatment of oral candidiasis, such as fluconazole and amphotericin B $[5,6]$.

Another aspect related to antifungal resistance and the recurrence of infection is the ability of Candida spp. to form biofilms on various surfaces in the oral cavity. A biofilm has been defined as a community of microorganisms organized at interfaces, enclosed in a self-produced polymeric matrix and adhered to an inert or living tissue. The presence of an exopolymeric matrix couple with the organization of layers of cells may confer protection of organisms in the inner layers, thereby contributing to antifungal resistance [7-9]. For example, biofilms formed in vitro by $C$. albicans on silicone catheters were sensitive to micafungin only at a concentration 100 to 500 times higher than the minimum inhibitory concentration (MIC) in planktonic cells [10]. 
The search for alternative therapies for oral candidiasis is a necessity and the use of medicinal plants seems to be one of the promising solutions [11,12]. The plant Melaleuca alternifolia has been used as an antiseptic remedy for decades. Although there is no published documentation of specific medicinal applications for $M$. alternifolia by Aboriginals prior to the colonization of Australia, the Bundjalung Aboriginals of New South Wales used the plant for medicinal purposes and spoke of the wound healing properties of the water from a lake into which $M$. alternifolia leaves had fallen [13]. The essential oil of $M$. alternifolia, termed tea tree oil, contains almost hundreds components, the majority of which are monoterpenes and related alcohols. It has a minimum content of $30 \%$ of terpinen-4-ol and a maximum content of $15 \%$ of 1,8 -cineole. Terpinen-4-ol is a major M. alternifolia component and exhibits strong antimicrobial and anti-inflammatory properties, whereas 1,8-cineole is probably an undesirable allergen in $M$. alternifolia products [14]. Preliminary trials suggest that $M$. alternifolia formulations may be effective in the treatment of acne and fungal infections, and in bacterial pathogen decolonization protocols [13]. The antimicrobial activity of $M$. alternifolia is attributed to its ability to denature proteins and alter the properties and function of the cell wall membrane, leading to the loss of intracellular components and eventually cell death [15-17].

Several in vitro studies described the antifungal activity of $M$. alternifolia against Candida isolates in planktonic growth, with MICs typically between $0.5-2 \%$ (v/v) [17] and showed inhibition of germ tube formation by $C$. albicans [18]. Few studies were performed in vivo to confirm and strengthen the in vitro results $[19,20]$. Because $M$. alternifolia shows promise as a topical anti-candidal therapy and its antimicrobial effects depend on the concentration used [17], the aim of this study was to determine the minimal concentration of $M$. alternifolia required to eradicate C. albicans biofilms formed in vitro and to study the effects of this concentration in treating oral candidiasis induced in an immunosuppressed mouse model.

\section{Methods}

\section{Microbial strains and culture conditions}

C. albicans strain ATCC 18804 was used for all experimental assays in this study. This strain was stored as frozen stocks in $30 \%$ glycerol at $-80^{\circ} \mathrm{C}$, subcultured on Sabouraud Dextrose agar plates and routinely grown in Sabouraud liquid medium at $37^{\circ} \mathrm{C}$.

\section{M. alternifolia essential oil}

M. alternifolia was purchased at the Federal University of Viçosa/UFV. The UFV has the botanical identification of the plant studied, as well as its own deposit in a herbarium voucher specimen of the University, with registration number 30839 corresponding to $M$. alternifolia. The oil was obtained by the steam distillation technique. The oil sample contained the following major components: terpinen-4-ol (42.8\%), $\gamma$-terpinene $(20.4 \%), p$-cymene (9.6\%), $\alpha$-terpinene (7.9\%), 1,8-cineole (3\%), $\alpha$-terpineol $(2,8 \%)$ and $\alpha$-pinene $(2.4 \%)$ as determined by gas chromatography mass spectrometry [21].

\section{In vitro activity of $M$. alternifolia essential oil: Determination of Minimal Inhibitory Concentration (MIC) in planktonic culture}

The MIC of M. alternifolia was determined by the broth microdilution method according to the Clinical and Laboratory Standards Institute (CLSI) [22] document M27A2. Initially, C. albicans were grown in Sabouraud dextrose agar for $48 \mathrm{~h}$ at $37^{\circ} \mathrm{C}$. Then, the yeast suspension was prepared in $5 \mathrm{~mL}$ sterile saline $(0.85 \%)$ and the cellular density was adjusted to 0.284 using a spectrophotometer (B582, Micronal, São Paulo, Brazil) at wavelength $=530 \mathrm{~nm}$, resulting in a standard solution with $1 \times 10^{6}$ cells $/ \mathrm{mL}$. The standard solution was diluted 1:50 in RPMI 1640 medium with L-glutamine without sodium bicarbonate and buffered with 0.165 M morpholinepropanesulfonic acid (MOPS; SigmaAldrich, Steinheim, Germany), followed by a 1:20 dilution to obtain a final concentration of $1-5 \times 10^{3}$ cells $/ \mathrm{mL}$.

Eleven serial 1:2 dilutions were made from the $M$. alternifolia into a 96-well plate (25 to $0.01 \%(\mathrm{v} / \mathrm{v})$ ) from $100 \mu \mathrm{L}$ of $M$. alternifolia in $100 \mu \mathrm{L}$ of culture medium RPMI 1640 at pH 7.0 \pm 0.1 . Subsequently, $100 \mu \mathrm{L}$ of the standardized suspension of $C$. albicans were added to each well of 96-well plate. A well for positive control (medium with inoculum) and another well for negative control (medium alone) were included.

Tween-80 (final concentration $0.001 \% \mathrm{v} / \mathrm{v}$ ) was included to facilitate oil solubility [19]. The plates were incubated at $37^{\circ} \mathrm{C}$ for $24 \mathrm{~h}$. Minimal inhibitory concentration (MIC) was determined in the well of lowest concentration, in which turbidity was not observed when compared to oilfree growth control. This experiment was performed independently in triplicate.

\section{In vitro activity of $M$. alternifolia essential oil: Determination of Minimal Biofilm Eradication Concentration}

C. albicans biofilms were formed in vitro using the methodology described by Seneviratne et al. [23] with some modifications. Cultures of C. albicans grown on Sabouraud dextrose agar (Himedia) at $37^{\circ} \mathrm{C}$ for $18 \mathrm{~h}$ were harvested in yeast nitrogen base (YNB, Himedia) supplemented with 50 $\mathrm{mM}$ glucose (Vetec, Duque de Caxias, RJ, Brazil). After an $18 \mathrm{~h}$ incubation at $37^{\circ} \mathrm{C}$, yeasts were centrifuged at $358 \times g$ for 10 minutes, washed twice with PBS, resuspended with YNB supplemented with $100 \mathrm{mM}$ glucose and adjusted to an optical density of 0.381 at $530 \mathrm{~nm}\left(10^{7}\right.$ cells $\left./ \mathrm{mL}\right)$ using a 
spectrophotometer (B582, Micronal). A $250 \mu \mathrm{L}$ aliquot of C. albicans suspension was pipetted into each well of a 96well flat-bottom microtiter plate (Costa Corning). The plate was incubated for $90 \mathrm{~min}$ at $37^{\circ} \mathrm{C}$ in a shaker at $75 \mathrm{rpm}$ (Quimis, Diadema, Brazil) for the initial adhesion phase. After this period, the wells were washed with $250 \mu \mathrm{L}$ of PBS to removed loosely adherent cells. A $250 \mu \mathrm{L}$ aliquot of YNB supplemented with $100 \mathrm{mM}$ glucose was then pipetted into each washed well, and the plates were incubated at $37^{\circ} \mathrm{C}$ in a shaker at $75 \mathrm{rpm}$ for $48 \mathrm{~h}$. The broth was changed every $24 \mathrm{~h}$. Plates with biofilms formed by $C$. albicans were washed with $250 \mu \mathrm{L}$ of PBS to remove loosely adherent cells.

The biofilm formed in each well was immersed in 250 $\mu \mathrm{L}$ of $M$. alternifolia suspended in $1 \%$ Tween 80 for 5 min in an orbital shaker (Solab, Piracibaca, Brazil). The concentration of $M$. alternifolia tested ranged from the MIC to $25 \%$. The biofilm of the control group was pipetted with phosphate buffered saline (PBS) for the same period of time. All tests were performed in triplicate.

After treatments with $M$. alternifolia, biofilm cells were scraped off the well wall using a sterile toothpick and transferred to Falcon tubes containing $10 \mathrm{~mL}$ PBS. To disrupt the biofilms, the contents of the tubes were homogenized for $30 \mathrm{~s}$ using an ultrasonic homogenizer (Sonoplus HD 2200, Badelin Electronic, Berlim, Germany) with an output power of $50 \mathrm{~W}$. The solution in the Falcon tubes was considered to be diluted by a factor of $10^{-1}$. Serial dilutions were then made using each original $10^{-1}$ dilution, and aliquots of $0.1 \mathrm{~mL}$ were seeded onto Sabouraud dextrose agar (Himedia) plates that were incubated at $37^{\circ} \mathrm{C}$ for $48 \mathrm{~h}$. After the incubation period, $\mathrm{CFU} / \mathrm{mL}$ values were determined for each plate. Minimal biofilm eradication concentration was defined as the lowest concentration of oil that resulted in complete inhibition of CFU/mL.

\section{Analysis of $C$. albicans biofilms formed in vitro by Scanning Electron Microscopy (SEM)}

Biofilms of $C$. albicans treated with the minimal biofilm eradication concentration of TTO $(n=2)$ and treated with PBS as a control group $(n=2)$ were subjected to SEM analysis.

Biofilms were formed and treated by $M$. alternifolia as described above with a minor modification: biofilms were formed on polystyrene discs approximately $8 \mathrm{~mm}$ in diameter that had been previously sterilized in a $20-\mathrm{kGy}$ gamma radiation chamber (cobalt 60) for $6 \mathrm{~h}$ (Embrarad, São Paulo, Brazil). The discs were placed into 24-well plates (Costa Corning, New York, USA) in which the total volume of suspension, PBS, broth culture and essential oil was $1 \mathrm{~mL}$. The discs were transferred after biofilm formation to 24-well plates fixed in $2.5 \%$ glutaraldehyde for $1 \mathrm{~h}$ and dehydrated in several ethanol washes (10, 25, 50, 75 and $90 \%$ for $20 \mathrm{~min}$ each and $100 \%$ for $60 \mathrm{~min}$ ). The plates were then incubated at $37^{\circ} \mathrm{C}$ for $24 \mathrm{~h}$ to dry the discs. The discs were transferred to aluminium stubs and covered with gold for $120 \mathrm{~s}$ at $40 \mathrm{~mA}$ (BAL-TEC 50D 050 Sputter Coater, Liechtenstein). After metallization, the biofilms were examined and photographed by SEM (Jeol JSM 5600, Tokyo, Japan) operating at $15 \mathrm{kV}$ in increments of 1000 and 5000 times.

\section{In vivo activity of $M$. alternifolia essential oil in oral candidiasis induced in an immunosuppressed mouse model}

All animal experiments were conducted in accordance with the policies for animal care, welfare, and use of the Institute of Science and Technology/UNESP and to the Declaration of Helsinki. This study was approved by the Research Ethics Committee of the Institute of Science and Technology/UNESP (protocol number 024/2009PA/CEP). Twenty-four adult male mice (Mus musculus, Albinus, Swiss) with twelve-week-old that tested negative for the Candida genus in the oral cavity, weighing approximately 30 to $60 \mathrm{~g}$, were included in the study. Animals were divided into 2 groups: treated with TTO $(\mathrm{n}=12)$ and treated with physiological solution $(\mathrm{n}=12)$. In each group, 10 mice were used for analysis by optical microscopy and 2 mice were used for scanning electron microscopy. The design of the experiments is shown in Table 1.

\section{Induction of experimental candidiasis}

The methodology described by Takakura et al. [24] was used to induce experimental candidiasis with some modifications. Briefly, animals were immunosuppressed with 2 subcutaneous injections of prednisolone (Depo-Medrol, laboratórios Pfizer Ltda., Guarulhos, SP, Brazil) at a dose of $100 \mathrm{mg} / \mathrm{kg}$ of body weight 1 day before and 3 days after infection with Candida. Tetracycline chloride (Terramicina, Laboratórios Pfizer Ltda., Guarulhos, SP, Brazil) was

\section{Table 1 Design of the study of in vivo activity of} M. alternifolia essential oil

\begin{tabular}{ll}
\hline $\begin{array}{l}\text { Day of } \\
\text { Experiment }\end{array}$ & Methodology \\
\hline Day 1 & $1^{\circ}$ injection of prednisolone \\
Day 2 & Inoculation of C. albicans in the oral cavity of mice \\
Day 5 & $2^{\circ}$ injection of prednisolone \\
Day 6 & Treatment of oral candidiasis with M. alternifolia \\
& essential oil or physiological solution (control group) \\
& $\begin{array}{l}\text { Recovery of C. albicans from the tongue dorsum } \\
\text { of mice before and immediately after } \\
\text { experimental treatment } \\
\text { Euthanasia of the mice: macroscopic analysis, optical } \\
\text { microscopy, and Scanning Electron Microscopy } \\
\text { (SEM) of the tongue dorsum of mice. }\end{array}$ \\
\hline
\end{tabular}


administered in the drinking water at a concentration of $0.83 \mathrm{mg} / \mathrm{mL}$ beginning 1 day before infection and was maintained throughout the experiment. A $50 \mu \mathrm{L}$ intramuscular injection of chlorpromazine chloride $(10 \mathrm{mg} / \mathrm{kg}$ of body weight; Amplictil, Sanofi Aventis, Suzano, SP, Brazil) in each thigh was used to sedate the animals.

C. albicans grown for $24 \mathrm{~h}$ at $37^{\circ} \mathrm{C}$ on Sabouraud dextrose agar (Himedia, Mumbai, Maharashtra, India) was resuspended in $10 \mathrm{~mL}$ PBS and centrifuged at $358 \mathrm{xg}$ for 10 minutes. The pellet was resuspended in $10 \mathrm{~mL}$ PBS and adjusted to $10^{8}$ cells $/ \mathrm{mL}$ after counting in a Neubauer chamber (Laboroptik GMBH, Bad Homburg, Germany). A sterile swab (Absorve, Cral, São Paulo, SP, Brazil) soaked in the $C$. albicans suspension was used to inoculate the sedated mice by rubbing the swab for 1 minute on the tongue dorsum.

\section{Treatment of experimental oral candidiasis with $M$. alternifolia essential oil}

At 4 days post C. albicans inoculation, animals were anesthetized by intramuscular injection of ketamine (União Química, São Paulo, Brazil) at a concentration of $100 \mathrm{mg} / \mathrm{kg}$ of body weight and Xylazine (Produtos Veterinários J. A. Ltda., Patrocínio Paulista, SP, Brazil) at a dose of $10 \mathrm{mg} / \mathrm{kg}$ body weight. The minimal biofilm eradication concentration of $M$. alternifolia determined by the in vitro assay (12.5\%) was used to treat oral candidiasis. M. alternifolia was suspended in $1 \%$ Tween 80 and pipetted onto the dorsum of the tongue in $50 \mu \mathrm{L}$ for 3 times in intervals of 10 minutes. The control group received physiological solution in the same volume and with the same frequency.

\section{Recovery of $C$. albicans from the tongue dorsum of mice}

Samples from the tongue dorsum were collected with a mini-swab before and immediately after each experimental treatment, the swab was placed in a test tube containing $0.99 \mathrm{~mL}$ PBS and shaken for 1 minute. This solution was estimated to be diluted by a factor of $10^{-2}$ Candida from the soaked swab. Serial dilutions were subsequently made, and $0.1 \mathrm{~mL}$ of each dilution was plated onto the surface of Sabouraud dextrose agar (Himedia) containing chloramphenicol (Viximicina, São Paulo, SP, Brazil). Dilutions were plated in duplicate and incubated at $37^{\circ} \mathrm{C}$ for 48 hours. Candida colonies were counted on plates exhibiting 30 to 300 colonies to determine colony-forming units $(\mathrm{CFU}) / \mathrm{mL}$. Plates with fewer than 30 colonies from the initial $10^{-2}$ dilution were estimated to contain $10^{-1}$ Candida cells.

\section{Euthanasia of the mice}

One day after the experimental treatment, an excessive dose of anesthetic, $100 \mathrm{mg} / \mathrm{kg}$ body weight ketamine (União Química Farmacêutica Nacional S/A., Embu-Guaçu, SP,
Brazil) and $10 \mathrm{mg} / \mathrm{kg}$ body weight xylazine (Produtos Veterinários J. A. Ltda., Patrocínio Paulista, SP, Brazil) was administered to kill the mice, corresponding to 5 days after experimental candidiasis induction. Tongues were removed for macroscopic and microscopic analyses (optical and scanning electron microscopy, respectively).

\section{Macroscopic analysis of candidiasis on the tongue dorsum of mice}

Characteristic lesions of candidiasis on the tongue dorsum were observed using a stereomicroscope (Zeiss, Göttingen, Germany). For quantification of lesions on the tongue dorsum, scores were assigned from 0 to 4 : 0 , normal; 1 , white patches on less than $20 \%$ of the surface; 2 , white patches covering between $21 \%$ and $90 \%$ of the surface; 3 , white patches on more than $91 \%$ of the surface; and 4, thick white patchy pseudomembranes covering more than $91 \%$ of the surface [24].

\section{Optical microscopy of the tongue dorsum of mice}

For microscopic analysis of the lesions, the tongues were fixed in $10 \%$ formalin for $24 \mathrm{~h}$. After embedding in paraffin, $5 \mu \mathrm{m}$-thick tissue slices were cut and stained with hematoxylin-eosin (H\&E) and periodic acid-Schiff (PAS). The presence of candidiasis was analyzed using optical microscopy (Olympus, CX41, Toquio, Japan) at X400 magnification.

Candidiasis lesions were quantified by counting the number of hyphae and epithelial lesions in histological sections stained with PAS and H\&E, respectively. For each stain, two histological sections were selected randomly and analyzed from each animal. In each histological section, 21 histologic fields were analyzed in the anteroposterior direction in 42 histologic fields.

The presence of yeasts and hyphae was quantified according to the methodology of Junqueira et al. [25], attributing the following scores to histologic fields: 1,1 to 5 yeasts/hyphae; 2, 6 to 15 yeasts/hyphae; 3, 16 to 50 yeasts/hyphae; and 4, more than 50 yeasts/hyphae. For statistical analysis, a median of the scores obtained from the 42 histologic fields was determined per animal.

The intensity of the tissue lesions was evaluated by counting the number of histologic fields with the presence of epithelial lesions, such as epithelial hyperplasia, disorganization of the basal cell layer, exocytosis, spongiosis, loss of filiform papillae, hyperkeratosis and development of intraepithelial microabscesses. The mean of the number of histologic sections with epithelial lesions was determined per animal for statistical analysis.

\section{Scanning electron microscopy (SEM) of the tongue dorsum of mice}

For SEM analysis, tongues were fixed in $2.5 \%$ glutaraldehyde in phosphate buffer $(0.1 \mathrm{~mol} / \mathrm{L}$ and $\mathrm{pH} 7.3)$ for 24 
$\mathrm{h}$ at $4^{\circ} \mathrm{C}$. The tongues were washed with a physiological salt solution $(0.85 \% \mathrm{NaCl})$ for 30 minutes. Specimens were subsequently dehydrated in a series of ethanol solutions $(50 \%, 70 \%$, and $90 \%$ for 20 minutes each and $100 \%$ for 20 minutes 3 times). After dehydration, tongues were dried to the critical point using $\mathrm{CO}_{2}$ (Denton Vacuum DCP 1, Moorestown, NJ). The tongues were then fixed on aluminum stubs and coated with gold for 120 seconds at $40 \mathrm{~mA}$ (BAL-TEC 50 D 050 Sputter Coater, Liechtenstein) and evaluated using SEM (JEOL JSM 5600, Tóquio, Japan) at $15 \mathrm{kV}$. The images obtained from SEM analyses were evaluated only to identify yeasts, hyphae and tissue damage that characterize the experimental candidiasis induced in immunosuppressed mouse model. No quantification of Candida or epithelial lesions was performed.

\section{Statistical analysis of the data obtained from}

\section{experimental candidiasis}

The data obtained from the recovered CFU/mL and quantification of epithelial lesions using optical microscopy were analyzed by Student's $t$ test. The scores from the macroscopic analysis and the quantification of yeasts and hyphae in optical microscopy were evaluated using the non-parametric Mann-Whitney test. A $P$ value less than 0.05 was considered statistically significant.

\section{Results}

\section{In vitro activity of $M$. alternifolia essential oil}

The effects of $M$. alternifolia on the in vitro growth of C. albicans were first examined following the CLSI. Among the tested concentrations of M. alternifolia $(0.01$ to $25 \%$ ), the MIC value was determined to be $0.195 \%$ $(1.95 \mathrm{mg} / \mathrm{mL})$. After determination of the MIC, concentrations of $M$. alternifolia ranging from 0.195 to $25 \%$ were tested on $C$. albicans biofilms, and $12.5 \%$ was determined to be the minimal biofilm erradication concentration. To confirm complete inhibition of C. albicans biofilms after treatment with $12.5 \% M$. alternifolia, the biofilms were analyzed by scanning electron microscopy (SEM). In SEM analysis, no formation of hyphae or blastoconidia was observed in the biofilms treated with M. alternifolia. However, a heterogeneous biofilm composed of blastoconidia, pseudohyphae and hyphae was observed on the polystyrene discs in the control group without $M$. alternifolia treatment (Figure 1).

\section{In vivo activity of $M$. alternifolia essential oil}

The numbers of $C$. albicans recovered from the oral cavity of mice before and immediately after experimental treatments with $M$. alternifolia or physiological solution (control group) are shown in Figure 2. The group treated with $M$. alternifolia showed a $5.33 \log _{10}$ reduction in the number of $C$. albicans cells after treatment, while

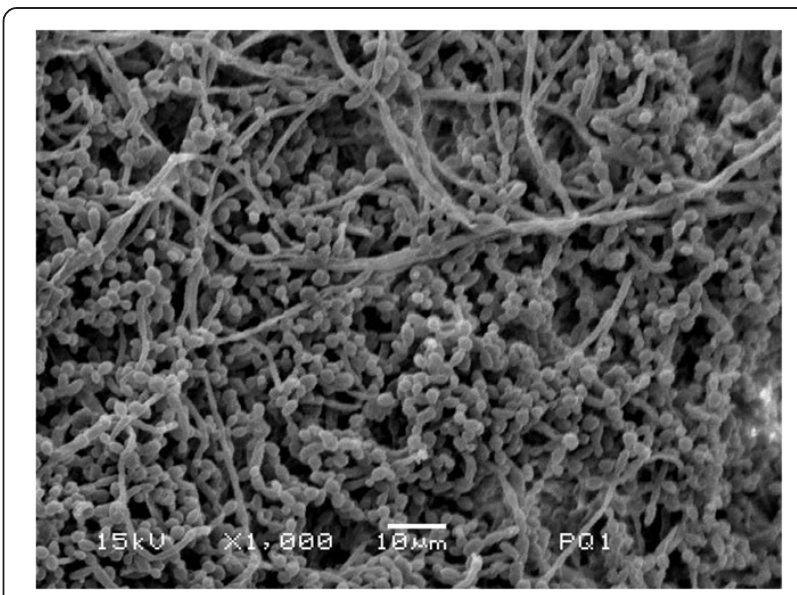

Figure 1 Scanning electron microscopy (SEM) of C. albicans biofilms formed in vitro on the polystyrene discs. The biofilm formed after $48 \mathrm{~h}$ was composed of blastoconidia, pseudohyphae and hyphae (Control group).

the control group had a $0.24 \log _{10}$ reduction after application of physiological solution, indicating that the essential oil of $M$. alternifolia significantly reduced the colonization by $C$. albicans in the oral cavity of mice.

After $24 \mathrm{~h}$ of experimental treatments, macroscopic analysis of the tongue dorsum showed the presence of candidiasis lesions characterized by pseudomembranous white plaques (Figure 3) in both experimental groups (treated with $M$. alternifolia and control) with a median

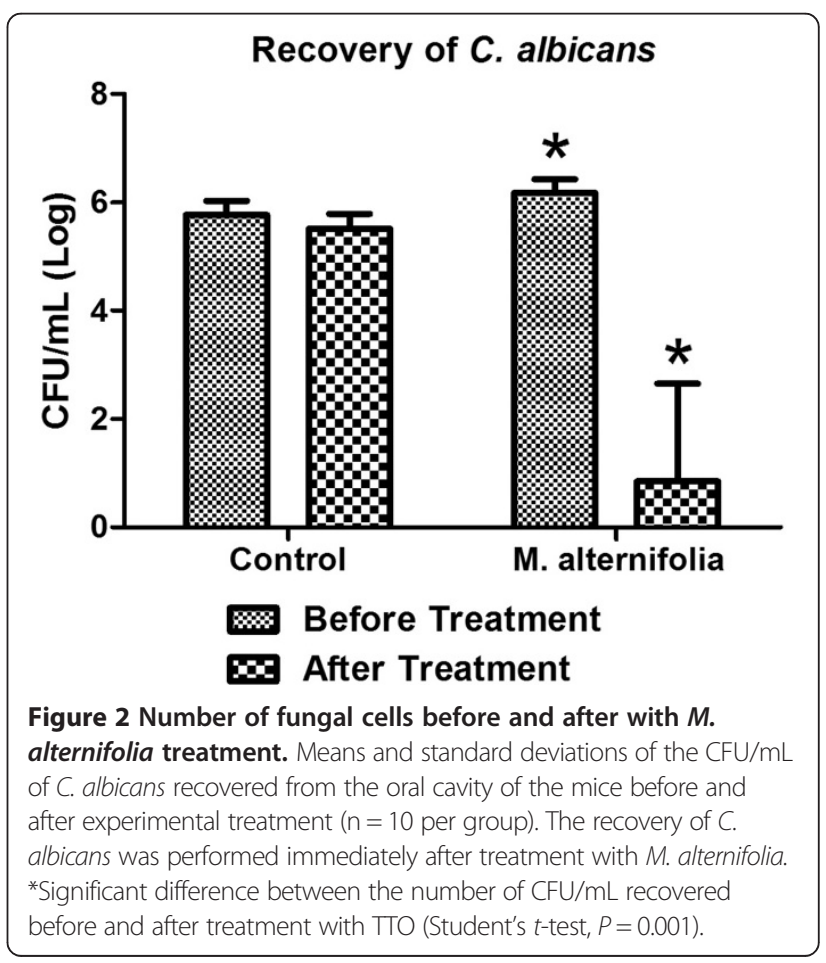




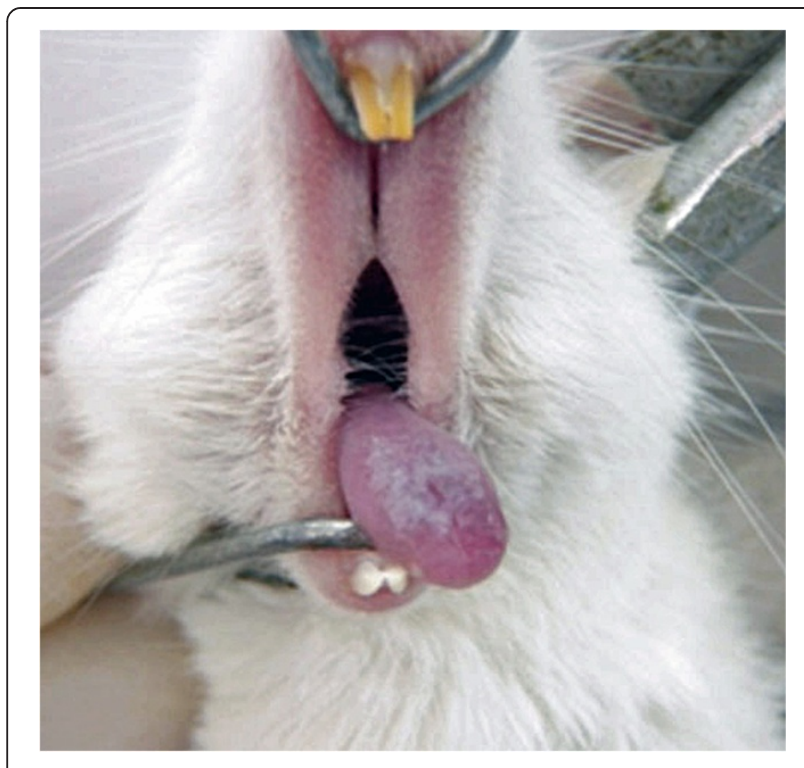

Figure 3 Macroscopic lesions of candidiasis on the tongue dorsum characterized by pseudomembranous white plaques.

score of 2, representing white patches covering between $21 \%$ and $90 \%$ of the tongue surface. In all mice studied, only scores 1 and 2 were identified. Although the number of animals receiving score 2 in the group treated with $M$. alternifolia was lower when compared to the control group, this difference was not significant (Figure 4). These data demonstrated that candidiasis lesions persisted for 24 $\mathrm{h}$ after treatment with essential oil of $M$. alternifolia.

In optical microscopy analysis, candidiasis lesions were represented by the presence of yeasts and hyphae limited to the keratinized layer on the tongue dorsum (Figure 5). In these regions, the epithelial tissue showed loss of filiform papillae, microabscesses, exocytosis, spongiosis, basal layer disorganization, epithelial hyperplasia, and an increased number of mitoses in the basal layer. Muscle inflammation, inflammatory infiltrate and some congested blood vessels in the lamina propria were also observed.

The hyphae and yeasts were quantified in histological sections stained by PAS (Figure 6). Although the median scores obtained for the M. alternifolia and control groups were scored 1 and 2 , respectively, no statistically significant difference was observed between groups treated with $M$. alternifolia and control $(P=0.2596)$. The epithelial lesions were also quantified using histological sections stained by H\&E (Figure 7), and it was observed that the group treated with $M$. alternifolia showed fewer candidiasis lesions when compared to the control group, this difference was statistically significant $(P=0.005)$.

SEM analysis on the tongue dorsum showed a large quantity of bacteria, yeasts and hyphae. Yeasts appeared in less quantity than hyphae and some hyphae were penetrating the epithelial tissue (Figure 8). In some regions, the

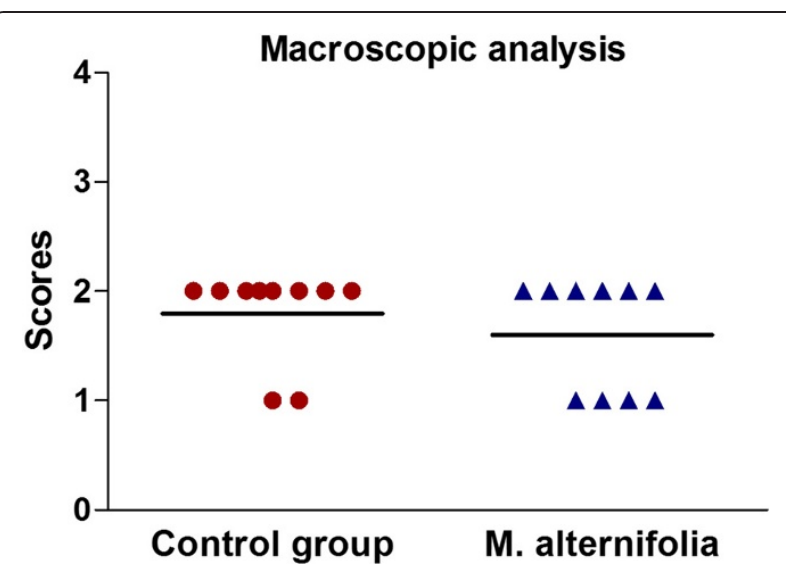

Figure 4 Macroscopic analysis of candidiasis on the tongue dorsum of mice. Scores obtained from the macroscopic analysis of candidiasis on the tongue dorsum of mice after $24 \mathrm{~h}$ of the treatment with essential oil of $M$. alternifolia or physiological solution for the control group ( $n=10$ per group). The scores were attributed according to Takakura el al. 2003 as follows: Score 0 (normal), Score 1 (white patches on less than $20 \%$ of the surface), Score 2 (white patches covering between $21 \%$ and $90 \%$ of the surface), Score 3 (white patches on more than $91 \%$ of the surface) and Score 4 (thick white patchy pseudomembranes covering more than $91 \%$ of the surface). There were no significant differences between the groups treated with M. alternifolia oil and control (Mann-Whitney test, $P=0.3662$ ).

filiform papillae were damaged, while papillae were lost in others, showing that the animals developed well-established candidiasis lesions on the tongue dorsum.

\section{Discussion}

Numerous essential oils such as M. alternifolia oil have been tested for both their in vitro and in vivo antifungal activity. M. alternifolia has been traditionally used by Australian natives and more recently, it has become a popular essential oil used as a non-ethnic remedy worldwide $[12,20]$. In this study, we demonstrated that the essential oil of $M$. alternifolia at a $12.5 \%$ concentration completely inhibited the $C$. albicans biofilms formed in vitro and had a protective effect against oral C. albicans infections in mice.

M. alternifolia has demonstrated activity against C. albicans [17-20]. New studies that can identify and evaluate the clinical effectiveness of $M$. alternifolia need be developed, such as assays with different experimental models and cell lines by evaluating the cytotoxicity, various concentrations and combinations with conventional antifungal agents to verify the possible synergism. Our findings can contribute in the future to prove M. alternifolia as an adjuvant clinical therapy, especially in cases of Candida lesions that is the most frequent fungal condition among humans living with HIV.

First, the in vitro effects of $M$. alternifolia on $C$. albicans were examined by the broth microdilution method 

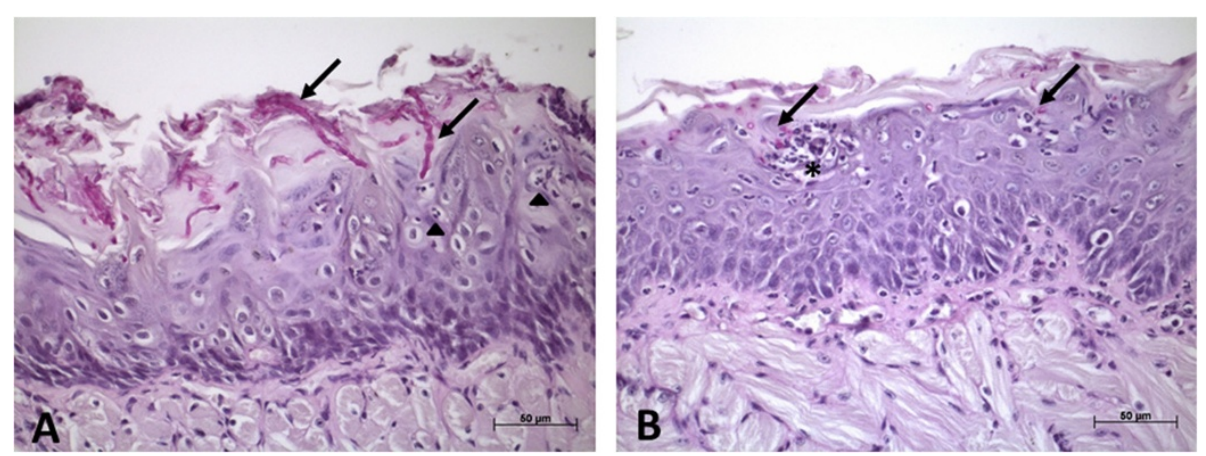

Figure 5 Sagittal section of the dorsum of the tongue of mice. A) Control group - Hyphae of C. albicans in the keratin layer (arrows) and areas of exocytosis (arrow head). B) M. alternifolia group - Yeasts of $C$. albicans in the keratin layer (arrows) and intraepithelial microabscesses (*). PAS staining, 400X magnification.

(CLSI), and the MIC obtained was $0.195 \%(1.95 \mathrm{mg} / \mathrm{mL})$. According to Carson et al. [13], the MICs of M. alternifolia for yeasts generally range between 0.03 and $0.5 \%$, while fungicidal concentrations generally range from 0.12 to $2 \%$. In addition to inhibiting the growth of Candida, some studies have shown that $M$. alternifolia inhibits the formation of germ tubes and decreased the cell surface hydrophobicity of C. albicans [13,17,18]. Hammer et al. [18] verified that germ tube formation was affected by the presence of sub-inhibitory concentrations of $M$. alternifolia oil, and it was completely inhibited by the presence of $0.25 \%$ TTO. Sudjana et al. [17] investigated the effects of the oil from M. alternifolia on C. albicans cell adhesion to both abiotic and biotic surfaces. Adhesion of C. albicans to polystyrene was significantly reduced for 3 isolates at $0.031 \%, 6$ isolates at $0.062 \%$ and $0.125 \%$ and for all 7 isolates studied at $0.25 \%$ M. alternifolia. Similarly, adhesion to buccal epithelial was also significantly reduced in the presence of $0.016-0.062 \%$ M. alternifolia.

Essential oil mouthwashes containing a range of natural plant extracts, including thymol, eucalyptol, bioflavanoids and $M$. alternifolia oil derivatives, demonstrated direct bactericidal and anticandidal activity in vitro. It is thought that essential oil mouthwashes kill microorganisms by cell membrane disruption and enzyme inhibition. However, the effectiveness of natural antimicrobials on established
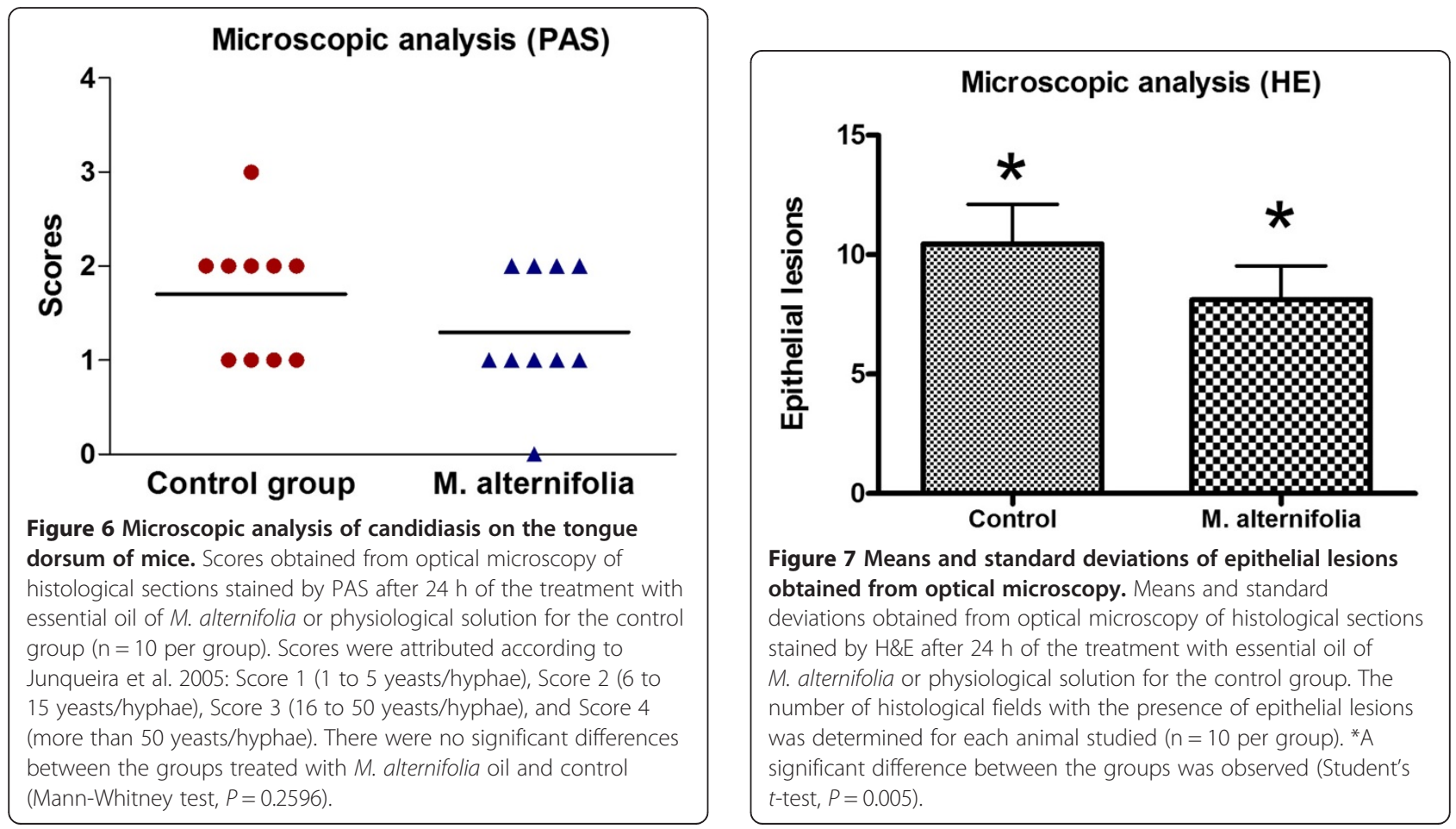

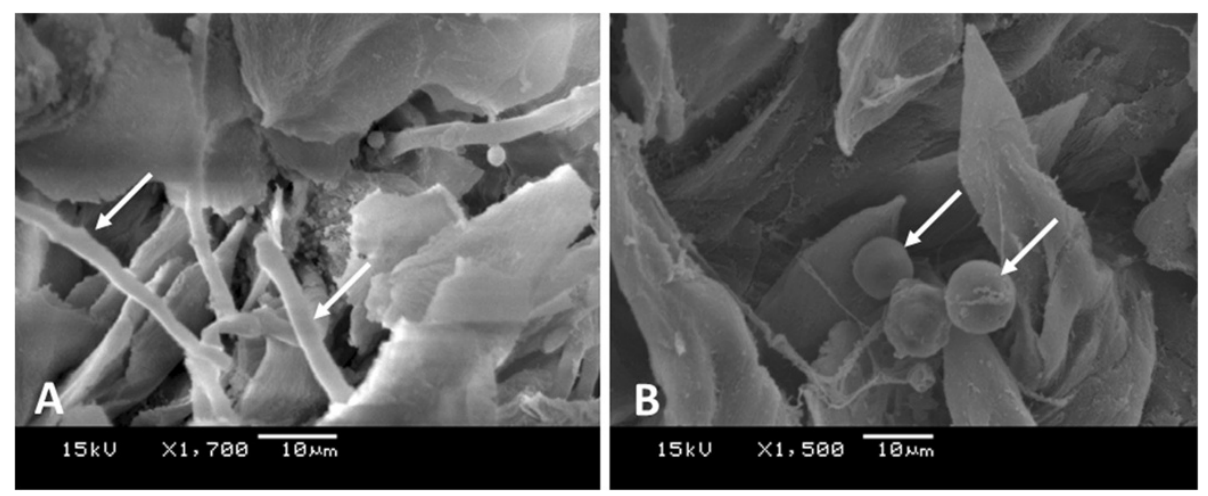

Figure 8 Scanning electron microscopy of the tongue dorsum. A) Control group - Hyphae of C. albicans (arrows) penetrating the tissue of the tongue dorsum after $24 \mathrm{~h}$ of the treatment with physiological solution. B) M. alternifolia group -Yeasts of C. albicans (arrows) on the tissue of the tongue dorsum after $24 \mathrm{~h}$ of the treatment with M. alternifolia oil.

biofilms in the oral cavity is unknown, with incomplete penetration by the agents being reported. The clinical efficacy of essential oil mouthwashes has been studied, but largely against plaque bacteria. Therefore, the clinical benefits of these agents in treating oral candidiasis remain to be established [1].

In this study, we determined the minimal concentration of M. alternifolia oil necessary to eradicate C. albicans biofilms formed in vitro. The minimal biofilm eradication concentration (MBEC) was $12.5 \%$, equivalent to 64 times the MIC. Budzynska et al. [11] also evaluated the minimal inhibitory concentration (MIC) and the minimal biofilm eradication concentration (MBEC) of $M$. alternifolia in biofilms formed by Staphylococcus aureus. The MIC value was $0.38 \%$, and the authors achieved an MBEC concentration of 173 times the MIC for $1 \mathrm{~h}$ of treatment, whereas 8 times the MIC was sufficient to obtain a $90 \%$ reduction in biomass metabolic activity after $4 \mathrm{~h}$ of treatment.

After establishing the MBEC, we tested the in vivo effects of M. alternifolia at a $12.5 \%$ concentration on oral candidiasis. For this, we used an experimental model of oral candidiasis in immunosuppressed mice developed by Takakura et al. [24]; this model is an established methodology that is widely used by several authors for the study of this infection [20,26-29]. In the control group of this study, a large number of viable Candida cells (5-6 $\log _{10}$ ) were recovered from the oral cavity of mice. This finding is in agreement with studies by Costa et al. [28] and Ninomiya et al. [20] that recovered 5-6 $\log _{10}$ Candida cells from the oral cavity of animals.

In contrast, a relatively low number of $C$. albicans cells was recovered from the oral cavity of mice treated with $M$. alternifolia. The treatment with this essential oil resulted in a $5.33 \log _{10}$ reduction of C. albicans, indicating clinical efficacy of $M$. alternifolia for the treatment of oral candidiasis. Ninomiya et al. [20] also studied the effects of $M$. alternifolia on oral candidiasis in an immunosuppressed mouse model and found an approximate $1 \log _{10}$ reduction of $C$. albicans after treatment with $M$. alternifolia at concentrations of 1 and 4\%. Probably, the highest Candida reduction observed in our study was achieved by the concentration of $M$. alternifolia tested (12.5\%).

According to Hammer et al. [30], while the antimicrobial properties of $M$. alternifolia oil are increasingly well-characterized, relatively limited data are available on the safety and toxicity of this oil. Greay et al. [31] showed that topical treatments of $10 \%$ M. alternifolia had significant activity against subcutaneous tumours in immunocompetent mice. The antitumor effect of topical M. alternifolia was accompanied by skin irritation similar to other topical chemotherapeutic agents, but unlike other approved topical agents, this irritation was quickly resolved. Furthermore, topical $M$. alternifolia caused an influx of neutrophils in the treated area, with no evidence of systemic toxicity. The lack of systemic activity within the treatment period was verified by normal body weigth, no changes in serum alkaline phosphatase or aspartate aminotransferase and normal liver histology of the animals studied.

Clinical evidence suggests that topical use of the oil is relatively safe and that adverse events are minor, selflimiting and occasional [30]. In a study conducted by Veien et al. [32], 217 patients consecutively sampled in a dermatology clinic were patch-tested with $10 \% M$. alternifolia with no irritant reactions recorded. Catalán et al. [33] tested $20 \% \mathrm{M}$. alternifolia to identify the in vitro and in vivo activity of this oil mixed with different tissue conditioners on the $C$. albicans strain. In the in vitro study, these authors verified that Coe-Comfort or Fitt conditioners mixed with $M$. alternifolia exhibited total inhibition of $C$. albicans. Patients treated with $M$. alternifolia mixed with Coe-Comfort in an in vivo study 
showed a significant decrease in palatal inflammation when compared to those treated with Coe-Comfort alone. However, M. alternifolia can be toxic if ingested, as evidenced by studies with experimental animals and from cases of human poisoning $[34,35]$. The $50 \%$ lethal dose for M. alternifolia in a rat model is 1.9 to $2.6 \mathrm{~mL} / \mathrm{Kg}$. Incidences of oral poisoning in children and adults have been reported, and in all cases, patients responded to supportive care and recovered without apparent sequelae [13].

In this study, $M$. alternifolia was suspended in $1 \%$ Tween 80 to be applied on the dorsum of the tongue. Tween 80, a nonionic surfactant and an emulsifier, is frequently used as a carrier for M. alternifolia. Considering the biological effects of Tween 80 in experimental oral candidiasis treated by $M$. alternifolia, Ninomiya et al. $[20,36]$ used $1 \%$ Tween 80 as control group and found similar results of $C$. albicans $\mathrm{CFU} / \mathrm{mL}$ compared to other studies of oral candidiasis in immunosuppressed mice that used physiological solution as control group. Several studies showed that Tween 80 has low toxicity for human cells and experimental animals [37,38]. In addition, its application is permitted for intradermal and intravenous injection in human beings [38].

After verifying that $M$. alternifolia at concentration of $12.5 \%$ significantly reduced the C. albicans colonization in the oral cavity of mice, we tested the effects of $M$. alternifolia treatment on the candidiasis lesions and tissue repair. Therefore, mice were killed 24 hours after the treatment and the tongues were removed for macroscopic and microscopic analyses. In these analyses, we verified that the animals treated with $M$. alternifolia showed fewer candidiasis lesions when compared to untreated animals (control group). Despite this, no statistically significant difference was observed for the macroscopic analysis and quantification of hyphae (histological sections stained by PAS) between $M$. alternifolia and control-treated groups. Only the quantification of epithelial lesions (histological sections stained by $H \& E$ ) showed a statistically significant difference between groups.

However, Ninomiya et al. [20] found a significant reduction in the macroscopic lesions of the dorsum tongue for groups treated with $M$. alternifolia. This difference might be attributed to the time of $M$. alternifolia application in relation to the stage of candidiasis infection. Ninomiya et al. [20] applied M. alternifolia on the dorsum tongue at 3 and $24 \mathrm{~h}$ after infection by C. albicans. Because it is known that $C$. albicans form germ tubes and hyphae 3 hours after inoculation in experimental oral candidiasis [39], Ninomiya et al. [20] may have found a significant reduction in macroscopic lesions because $M$. alternifolia prevented Candida invasion of the epithelium by hyphae. These data suggest that early administration of $M$. alternifolia is highly effective. In the present study, M. alternifolia was applied 4 days after infection by $C$. albicans.
Therefore, oral candidiasis lesions were already wellestablished, and consequently impaired the action of $M$. alternifolia. Furthermore, we evaluated candidiasis lesions on the dorsum tongue $24 \mathrm{~h}$ after application of $M$. alternifolia, and this time period may not have been long enough for improvement of the lesions and tissue repair to occur.

\section{Conclusions}

In this study, we concluded that $M$. alternifolia oil at a $12.5 \%$ concentration was effective to eradicate a C. albicans biofilm formed in vitro and to reduce yeasts of C. albicans in an immunosuppressed mouse model.

\section{Competing interests}

The authors declare that they have no competing interests.

\section{Authors' contributions}

Conceived and designed the experiments: RDR, VMCR, ACBPC, CFA, PPB, ALA. Performed the experiments: JCJ, RDR, ACBPC, VMCR. Analyzed the data: $J C J, A C B P C, V M C R, A O C J, A L A$. Contributed reagents/materials/analysis tools: JCJ, AOCJ. Wrote the paper: RDR, JCJ, ALA, AOCJ. All authors read and approved the final manuscript.

\section{Acknowledgments}

The authors thank Prof. Antônio Lelis Pinheiro for his assistance with the preparation of M. alternifolia essential oil and Prof. Oslei Paes de Almeida and Adriano Luis Martins for their assistance with scanning electron microscopy of the dorsum tongue. We acknowledge the São Paulo Council of Research - FAPESP, Brazil (Grants 2010/19117-7, 2010/06602-4 and 2012/02184-9) for supporting this research.

Received: 29 April 2014 Accepted: 10 December 2014 Published: 15 December 2014

\section{References}

1. Williams DW, Kuriyama T, Silva S, Malic S, Lewis MA: Candida biofilms and oral candidosis: treatment and prevention. Periodontol 2000 2011, 55:250-265.

2. Naglik JR, Fidel PL Jr, Odds FC: Animal models of mucosal Candida infection. FEMS Microbiol Lett 2008, 283:129-139.

3. Souza RC, Junqueira JC, Rossoni RD, Pereira CA, Munin E, Jorge AO: Comparison of the photodynamic fungicidal efficacy of methylene blue, toluidine blue, malachite green and low-power laser irradiation alone against Candida albicans. Lasers Med Sci 2010, 25:385-389.

4. Junqueira JC, Vilela SF, Rossoni RD, Barbosa JO, Costa AC, Rasteiro VM, Suleiman JM, Jorge AO: Oral colonization by yeasts in HIV-positive patients in Brazil. Rev Inst Med Trop Sao Paulo 2012, 54:17-24.

5. Thompson GR 3rd, Patel PK, Kirkpatrick WR, Westbrook SD, Berg D, Erlandsen J, Redding SW, Patterson TF: Oropharyngeal candidiasis in the era of antiretroviral therapy. Oral Surg Oral Med Oral Pathol Oral Radiol Endod 2010, 109:488-495.

6. Schelenz S, Abdallah S, Gray G, Stubbings H, Gow I, Baker P, Hunter PR: Epidemiology of oral yeast colonization and infection in patients with hematological malignancies, head neck and solid tumors. J Oral Pathol Med 2011, 40:83-89.

7. Seneviratne CJ, Jin L, Samaranayake LP: Biofilm lifestyle of Candida: a mini review. Oral Dis 2008, 14:582-590.

8. Kuhn DM, George T, Chandra J, Mukherjee PK, Ghannoum MA: Antifungal susceptibility of Candida biofilms: unique efficacy of amphotericin B lipid formulations and echinocandins. Antimicrob Agents Chemother 2002, 46:1773-1780

9. Dovigo LN, Pavarina AC, Mima EG, Giampaolo ET, Vergani CE, Bagnato VS: Fungicidal effect of photodynamic therapy against fluconazole-resistant Candida albicans and Candida glabrata. Mycoses 2011, 54:123-130.

10. Cateau E, Berjeaud JM, Imbert C: Possible role of azole and echinocandin lock solutions in the control of Candida biofilms associated with silicone. Int J Antimicrob Agents 2011, 37:380-384. 
11. Budzyńska A, Wieckowska-Szakiel M, Sadowska B, Kalemba D, Rózalska B: Antibiofilm activity of selected plant essential oils and their major components. Pol J Microbiol 2011, 60:35-41.

12. Harris R: Progress with superficial mycoses using essential oils. The International Journal of Aromatherapy 2002, 12:83-91.

13. Carson CF, Hammer KA, Riley TV: Melaleuca alternifolia (Tea Tree) oil: a review of antimicrobial and other medicinal properties. Clin Microbiol Rev 2006, 19:50-62.

14. Pazyar N, Yaghoobi R, Bagherani N, Kazerouni A: A review of applications of tea tree oil in dermatology. Int J Dermatol 2013, 52:784-790.

15. Cox SD, Mann CM, Markham JL: Interactions between components of the essential oil of Melaleuca alternifolia. J Appl Microbiol 2001, 91:492-497.

16. Carson CF, Mee BJ, Riley TV: Mechanism of action of Melaleuca alternifolia (tea tree) oil on Staphylococcus aureus determined by time-kill, lysis, leakage, and salt tolerance assays and electron microscopy. Antimicrob Agents Chemother 2002, 46:1914-1920.

17. Sudjana AN, Carson CF, Carson KC, Riley TV, Hammer KA: Candida albicans adhesion to human epithelial cells and polystyrene and formation of biofilm is reduced by sub-inhibitory Melaleuca alternifolia (tea tree) essential oil. Med Mycol 2012, 50:863-870.

18. Hammer KA, Carson CF, Riley TV: Melaleuca alternifolia (tea tree) oil inhibits germ tube formation by Candida albicans. Med Mycol 2000, 38:355-362.

19. Mondello F, De Bernardis F, Girolamo A, Cassone A, Salvatore G: In vivo activity of terpinen-4-ol, the main bioactive component of Melaleuca alternifolia cheel (tea tree) oil against azole-susceptible and -resistant human pathogenic Candida species. BMC Infect Dis 2006, 6:158.

20. Ninomiya K, Maruyama N, Inoue S, Ishibashi H, Takizawa T, Oshima H, Abe S: The Essential oil of Melaleuca alternifolia (Tea Tree Oil) and its main component, terpinen-4-ol protect mice from experimental oral candidiasis. Biol Pharm Bull 2012, 35:861-865.

21. Pereira TS, Sant'Ana JR, Silva EL, Pinheiro AL, Castro-Prado MAC: In vitro genotoxicity of Melaleuca alternifolia essential oil in human lymphocytes. J Ethnopharmacol 2014, 151:852-857.

22. NCCLS (National Committee for Clinical Laboratory Standards): Reference method for broth dilution antifungal susceptibility testing of yeasts. Approved standard-second edition. M27-A2. Wayne, PA, USA: 2002.

23. Seneviratne CJ, Silva WJ, Jin $\sqcup$, Samaranayake $Y H$, Samaranayake LP: Architectural analysis, viability assessment and growth kinetics of Candida albicans and Candida glabrata biofilms. Arch Oral Biol 2009, 54:1052-1060

24. Takakura N, Sato Y, Ishibashi H, Oshima H, Uchida K, Yamaguchi H, Abe S: A novel murine model of oral candidiasis with local symptoms characteristic of oral thrush. Microbiol Immunol 2003, 47:321-326.

25. Junqueira JC, Colombo CE, Martins Jda S, Koga Ito CY, Carvalho YR, Jorge AO: Experimental candidosis and recovery of Candida albicans from the oral cavity of ovariectomized rats. Microbiol Immunol 2005, 49:199-207.

26. Kamagata-Kiyoura $Y$, Abe $S$, Yamaguchi H, Nitta T: Protective effects of human saliva on experimental murine oral candidiasis. $J$ Infect Chemother 2004, 10:253-255.

27. Hayama K, Ishibashi H, Kitadate K, Yamazaki M, Abe S: Therapeutic effect of oligonol, a low-molecular polyphenol formulation derived from lychee fruits on murine oral candidiasis. Nihon Ishinkin Gakkai Zasshi 2010, 51:137-142.

28. Costa AC, Campos Rasteiro VM, da Silva Hashimoto ES, Araújo CF, Pereira CA, Junqueira JC, Jorge AO: Effect of erythrosine- and LED-mediated photodynamic therapy on buccal candidiasis infection of immunosuppressed mice and Candida albicans adherence to buccal epithelial cells. Oral Surg Oral Med Oral Pathol Oral Radiol 2012, 114:67-74.

29. Costa AC, Pereira CA, Junqueira JC, Jorge AO: Recent mouse and rat methods for the study of experimental oral candidiasis. Virulence 2013, 4:391-399.

30. Hammer KA, Carson CF, Riley TV, Nielsen JB: A review of the toxicity of Melaleuca alternifolia (tea tree) oil. Food Chem Toxicol 2006, 44:616-625.

31. Greay SJ, Ireland DJ, Kissick HT: Inhibition of established subcutaneous murine tumour growth with topical Melaleuca alternifolia (tea tree) oil. Cancer Chemother Pharmacol 2010, 66:1095-1102.

32. Veien NK, Rosner K, Skovgaard GL: Is tea tree oil an important contact allergen? Contact Dermatitis 2004, 50:378-379.

33. Catalán A, Pacheco JG, Martínez A, Mondaca MA: In vitro and in vivo activity of Melaleuca alternifolia mixed with tissue conditioner on
Candida albicans. Oral Surg Oral Med Oral Pathol Oral Radiol Endod 2008, 105:327-332.

34. Morris MC, Donoghue A, Markowitz JÁ, Osterhoudt KC: Ingestion of tea tree oil (Melaleuca oil) by a 4-year-old boy. Pediatr Emerg Care 2003, 19:169-171.

35. Moss A: Tea tree oil poisoning. Med J Aust 1994, 160:236

36. Ninomiya K, Hayama K, Ishijima AS, Maruyama N, Irie H, Kurihara J, Abe S: Suppression of inflammatory reactions by Terpinen-4-ol, a main constituent of Tea Tree Oil, in a murine model of oral candidiasis and its suppressive activity to cytokine production of macrophages in vitro. Biol Pharm Bull 2013, 36:838-844.

37. Arechabala B, Coiffard C, Rivalland P, Coiffard $\sqcup$, de Roeck-Holtzhauer Y: Comparison of cytotoxicity of various surfactants tested on normal human fibroblast cultures using the neutral red test, MTT assay and LDH release. J Appl Toxicol 1999, 19:163-165.

38. Bulcão RP, de Freitas FA, Dallegrave E, Venturini CG, Baierle M, Durgante J, Sauer E, Cassini C, Cerski CT, Zielinsky P, Salvador M, Pohlmann AR, Guterres SS, Garcia SC: In vivo toxicological evaluation of polymeric nanocapsules after intradermal administration. Eur J Pharm Biopharm 2014, 86:167-177.

39. Hisajima T, Ishibashi H, Yamada T, Nishiyama Y, Yamaguchi H, Funakoshi K, Abe S: Invasion process of Candida albicans to tongue surface in early stages of experimental murine oral candidiasis. Med Mycol 2008 46:697-704

doi:10.1186/1472-6882-14-489

Cite this article as: de Campos Rasteiro et al: Essential oil of Melaleuca alternifolia for the treatment of oral candidiasis induced in an immunosuppressed mouse model. BMC Complementary and Alternative Medicine 2014 14:489.

\section{Submit your next manuscript to BioMed Central and take full advantage of:}

- Convenient online submission

- Thorough peer review

- No space constraints or color figure charges

- Immediate publication on acceptance

- Inclusion in PubMed, CAS, Scopus and Google Scholar

- Research which is freely available for redistribution 